\title{
Behavioral Determinants of Obesity: Research Findings and Policy Implications
}

\author{
Sandra G. Affenito, ${ }^{1}$ Debra L. Franko, ${ }^{2}$ Ruth H. Striegel-Moore, ${ }^{3}$ and Douglas Thompson ${ }^{4}$ \\ ${ }^{1}$ School of Health and Natural Sciences, University of Saint Joseph, West Hartford, CT 06117, USA \\ ${ }^{2}$ Department of Counseling and Applied Educational Psychology, Northeastern University, Boston, MA 02115-5000, USA \\ ${ }^{3}$ Department of Psychology, Wesleyan University, Middletown, CT 06457-0408, USA \\ ${ }^{4}$ Thompson Research Consulting, LLC, Chicago, IL 60601, USA \\ Correspondence should be addressed to Sandra G. Affenito, saffenito@usj.edu
}

Received 18 June 2012; Accepted 18 June 2012

Copyright ( $) 2012$ Sandra G. Affenito et al. This is an open access article distributed under the Creative Commons Attribution License, which permits unrestricted use, distribution, and reproduction in any medium, provided the original work is properly cited.

\section{Introduction}

Obesity is a public health concern worldwide associated with significant health risks and comorbid conditions $[1,2]$. During the years between 1980 and 2008, the international prevalence of obesity has increased twofold. In 1980, 5\% of men and $8 \%$ of women were obese, according to the body mass index (BMI) reference of $\geq 30 \mathrm{~kg} / \mathrm{m}^{2}$. Almost three decades later, $10 \%$ of men and $14 \%$ of women presented with obesity. Current databases document that more than half a billion adults worldwide are obese; more women than men are obese, with estimates of 297 million and 205 million, respectively [3]. Childhood obesity is increasing at a similar pace. In 1990, 4.2\% of children were overweight and obese, and this percentage increased to $6.7 \%$ in 2010. Of great concern is the forecasted number of children who are to be obese by the year 2020: an estimated $9.1 \%$ or 60 million children worldwide [4]. Moreover, given that childhood obesity has been shown to track into adulthood $[5,6]$ and has been lined with significant health-related conditions and psychological effects [7], obesity prevention is imperative. Understanding the determinants of obesity is crucial for informing and developing effective prevention efforts, which should be based on a scientific understanding of the multiple and complex risk factors for obesity. The etiology of obesity is multifaceted; there are factors from multiple contexts, and interactions between factors that led to obesity are not yet well understood [7]. Indeed, a comprehensive approach is needed to potentially reverse the global pandemic of obesity $[7,8]$.

\section{Ecological Approach to the Study of Obesity}

This special issues focuses primarily on the behavioural factors associated with obesity (dietary intake; physical activity) with consideration of the contextual factors including the home, school, and neighborhood environments, which can affect health. Successful models of addressing obesity have examined behaviours and the environment, with the goal of altering the obesogenic environment through public health strategies aimed at promoting healthful eating and physical activity [1,9]. The ecological model of obesity has been described as "multilevel (e.g., regions, nations, states, cities, and neighborhoods)," taking into consideration the "multistructural components (e.g., physical environment, socioeconomic status, and social capital)" and "multifactorial lifestyle behaviours (e.g., diet, physical activity, and stress)" at "multi-institutional (e.g., school, local government, family, and local agency)" levels. Moreover, this ecological approach accounts for the interrelationships among these influences to better understand and measure the behavioural factors that negatively influence weight [10].

\section{Behavioural Influences on Energy Intake and Energy Expenditure}

More recently, research has concentrated the behavioural influences on obesity, that is, specific behaviors that may contribute to weight gain through overeating or reduced physical activity and the mechanisms by which the environment can affect health. Review of the literature points to 
the specific eating behaviors that are associated with obesity. These obesity-promoting behaviours include frequent fast food consumption, eating occasions away from home, large portion sizes, high consumption of beverages high in sugar, and breakfast omission [11-17]. In addition to these behavioural factors, multistructural variables such as the physical environment and socioeconomic status have been shown to have a significant influence on food intake and energy expenditure [10,18]. Environmental influences consist of the proximity of large supermarkets, the concentration of fast food establishments and restaurants in any given area, the availability of recreational areas for physical activity, and socioeconomic status, each of which may favorably or negatively influence dietary behaviours and physical activity patterns [8, 19-22]. For example, large supermarkets provide a wide variety of healthful foods at reasonable prices, which may influence food purchasing behaviours [21] and thus the availability of more healthful foods at home [23]. Finally, there has been substantial interest in ensuring a healthful eating environment in schools and the important association between the home environment and nutrient intake [14, 24].

\section{Contributions of this Special Issue Relating to the Behavioural Determinants of Obesity}

As illustrated in the array of articles presented in this special issue, environmental and societal transitions including changing dietary practices, modernization of lifestyles, urbanization, and globalization of the food supply have influenced the increasing prevalence of obesity in developed and developing countries [6,25]. Using cross-sectional data from a national study, Z. Abdeen and colleagues (2012) observed that slightly more than $60 \%$ of Palestinians who were between the ages of 18 and 64 years were overweight $(38 \%)$ or obese $(24.4 \%)$, and obesity was prevalent in urban areas. While the study design did not permit the investigators to examine potential contributing factors for the prevalence of obesity the results illuminate the changes in food consumption and physical activity patterns which were associated with urbanization. Demographic variables including age, educational level, sex, and marital status were also associated with BMI. Relative to men, higher levels of obesity and lower physical activity levels were documented among less educated Palestinian women. The authors noted that low physical activity among females may be partly due to the lack of exercise facilities for girls and women in Palestine. Moreover, girls' attitudes and beliefs about sports and outdoor physical activity need to be taken into account when interpreting these findings. In developing countries, girls are more engaged in domestic chores and less in playground activities [6]. In addition to culture, individual level and social factors relating to meal planning may be important considerations when examining genderrelated differences in obesity. In fact, in this special issue, T. J. B. Dummer and collaborators (2012) observed among females that the time between adolescence and adulthood is a critical period for weight gain. In addition to the physiological changes which occur with maturation, young adulthood is a period when independence peaks in relation to food planning, purchasing, and preparation. Nutritional health has been shown to be associated with an individual's environment not only in terms of access to healthful foods through food purchasing and preparation, but also through opportunities for physical activity.

An emerging body of research focuses on the role of the built environment in influencing food choice and physical activity and, by extension, obesity. Factors of the built environment include proximity to grocery stores and fast food establishments, quality of schools, opportunities for social interactions with neighbors, and places for walking and other types of physical activities. Unfavorable built environments such as few parks or recreational facilities, low perceived neighborhood safety, increased access to restaurants, and convenience stores were associated with higher body mass index scores among adolescents, particularly those of low socioeconomic status [21]. In developed countries, socioeconomic status, income level, and education are inversely correlated with obesity $[18,22]$, whereas in developing countries, there is a high prevalence of obesity among those of higher economic status living in urban settings [6].

When examining the potential association between socioeconomic status, the built environment, fast food outlet exposures, and overweight, A. Lebel and colleagues (2012) observed that characteristics of one's residence, such as the percentage of fast food establishments, financial insecurity, and diversity in social status, accounted for an important component of the territorial distribution of overweight. Moreover, for urban women of low socioeconomic status, the number and proximity of fast food restaurants significantly predicted obesity risk. Fast food outlet exposure was also examined among adolescents by $\mathrm{T}$. Khan and co-investigators (2012) in the early childhood longitudinal study. Utilizing price data and contextual outlet density data, the findings revealed that higher fast food prices, higher median household income, and reduced access to fast food establishments were associated statistically with reduced intake of fast food among children (T. Khan et al., 2012). The findings of these studies (T. Khan et al. 2012; A. Lebel et al., 2012) point to the important role of the built environment and socioeconomic status (SES) in relation to food choice. Community-level interventions are necessary to ensure access to healthful foods and physical activity. Understanding the socioeconomic differences and relevant aspects of built environments can inform the development of programs aimed at reducing obesity.

The articles in this special issue point to a complex relationship between SES and obesity that needs to be better understood. Numerous reports [19-22], including articles in this special issue, have found associations between obesity and SES (or proxies for SES, e.g., education), but the mechanisms tying SES to obesity are not well understood. Interestingly, Z. Abdeen and colleagues (2012) found that the SES/obesity association varied by gender-high education was associated with greater waist-height ratios for men but the opposite was true for women. Z. Abdeen and investigators (2012) cite lack of exercise facilities as 
a potential contributing factor to obesity among women in Palestine. One wonders whether more highly educated Palestinian women may have better access to appropriate exercise facilities, facilitating decreased levels of obesity; lack of exercise might be a possible mechanism tying low SES to high rates of obesity. In addition to having better access to exercise facilities, the literature suggests that higher-SES individuals have access to better-quality food [22] - this is supported by T. Khan and colleagues' (2012) finding that higher-SES individuals consumed fast food less frequently and L. H. McArthur and co-authors' (2012) finding that cost was a barrier to healthy snacking. This suggests that higher SES individuals have greater resources to protect themselves from an obesogenic environment. Further studies are needed to elucidate the mechanisms tying SES to obesity. This question has profound policy implications, especially if public resources are required to overcome economic barriers to combating obesity.

Technology has enabled investigators to obtain data from all fifty-nine public and private schools in Alberta, Canada to employ a web-based method of surveillance of behavioural factors relating to weight and physical activity among students in grades seven to ten. K. E. Storey and colleagues (2012) found that nonoverweight adolescents consumed more healthful breakfast meals and snacks. These healthful eating habits were related to a higher consumption of carbohydrate and fibre, significantly less fat, and significantly greater activity levels compared with obese adolescents. Meal behaviours were also examined by location. While consuming food outside of the home eating environment has been associated with increase in weight $[14,18], \mathrm{K}$. E. Storey and investigators did not find significant differences between BMI classification and food intake away from home. Differences in variables studied, study design, and the types of food consumed away from home may have led to varying results. Thus, the association between eating behaviours and body weight is complex. A true understanding of the association between meal behaviours and weight status requires consideration of the potential mediating influence of physical activity or other food-related factors such as convenience, palatability, and reduced cost [10], which may affect intake.

In this special issue, L. H. McArthur and colleagues found that cost and availability were barriers to healthful snacking, but location did not influence snacking patterns of college students (2012) . Eating behaviors among youth are influenced by foods available not only at home, but also at school, where they spend a significant proportion of their time. These studies (L. H. McArthur et al., 2012; K. E. Storey et al., 2012) highlight the importance of the school environment, for both adolescents and college students, in terms of availability of healthful meals and snacks and providing programs which promote healthful eating and daily physical activity. In addition to a healthy school environment, ensuring that the home food environment provides healthful foods will assist with efforts to control body weight. In the health-is-power intervention study, T. A. Ledoux and colleagues (2012) found that home availability of fruits and vegetables and weekly stress were related to fruit and vegetable intake among African-American and Hispanic women, a segment of the population who is at risk for obesity. Since energy-controlled diets that are rich in fruits and vegetables may help to achieve and maintain weight loss, these findings are of importance and can inform nutrition education programs targeting this population.

While individual studies published in this special issue present data on specific correlates of obesity, taken together, the findings emphasize that obesity needs to be studied within the framework of the broader social and community contexts within which individual behaviours relating to dietary intake and physical activity occur. Targeting multiple lifestyle behaviours may be an effective means of preventing weight gain, rather than focusing on one specific determinant associated with excess energy status and weight [17]. In addition, these papers add to the growing body of evidence suggesting that healthy lifestyles can only be sustained if the environment and culture which support unhealthy lifestyles are addressed [9]. Unhealthful nutrition habits and reduced physical activity are likely to endure, increasing the risk among today's youth for obesity and chronic diseases in the future, unless public health initiatives are designed to be multilevel and take into consideration the multiple correlates that influence behaviours surrounding dietary intake and physical activity. Future studies need to be designed to evaluate the interactions among behavioural and environmental risk markers for obesity in order to advance prevention efforts and intervention programs.

\section{Sandra G. Affenito \\ Debra L. Franko \\ Ruth H. Striegel-Moore \\ Douglas Thompson}

\section{References}

[1] J. O. Hill, J. C. Peters, V. A. Catenacci, and H. R. Wyatt, International strategies to address obesity, Obesity Reviews, vol. 9, supplement1, pp. 41-47, 2008.

[2] 2009-2010 Report: International Association for the Study of Obesity, 2009, http://www.iaso.org/site_media/uploads/IASO_ Summary_Report_2009.pdf.

[3] World Health Organisation, WHO, Obesity and overweight (Factsheet), 2011, http://www.who.int/mediacentre/factsheets /fs311/en/.

[4] M. de Onis, M. Blössner, and E. Borghi, "Global prevalence and trends of overweight and obesity among preschool children," American Journal of Clinical Nutrition, vol. 92, no. 5, pp. 1257-1264, 2010.

[5] K. M. Herman, C. L. Craig, L. Gauvin, and P. T. Katzmarzyk, "Tracking of obesity and physical activity from childhood to adulthood: the Physical Activity Longitudinal Study," International Journal of Pediatric Obesity, vol. 4, no. 4, pp. 281-288, 2009.

[6] N. Gupta, K. Goel, P. Shah, and A. Misra, "Childhood obesity in developing countries: epidemiology, determinants, and prevention," Endocrine Reviews, vol. 33, no. 1, pp. 48-70, 2012.

[7] J. C. Han, D. A. Lawlor, and S. Y. Kimm, "Childhood obesity," The Lancet, vol. 375, no. 9727, pp. 1737-1748, 2010.

[8] D. B. Reed, P. J. Patterson, and N. Wasserman, "Obesity in rural youth: looking beyond nutrition and physical activity," 
Journal of Nutrition Education and Behavior, vol. 43, no. 5, pp. 401-408, 2011.

[9] N. S. Mitchell, V. A. Catenacci, H. R. Wyatt, and J. O. Hill, "Obesity: overview of an epidemic," Psychiatric Clinics of North America, vol. 34, pp. 717-732, 2011.

[10] T. Baranowski, K. W. Cullen, T. Nicklas, D. Thompson, and J. Baranowski, "Are current health behavioral change models helpful in guiding prevention of weight gain efforts?" Obesity Research, vol. 11, no. 1, supplement, pp. 23S-43S, 2003.

[11] M. Schmidt, S. G. Affenito, R. Striegel-Moore et al., "Fast-food intake and diet quality in black and white girls: the National Heart, Lung, and Blood Institute growth and health study," Archives of Pediatrics and Adolescent Medicine, vol. 159, no. 7, pp. 626-631, 2005.

[12] R. H. Striegel-Moore, D. Thompson, S. G. Affenito et al., "Correlates of beverage intake in adolescent girls: the National Heart, Lung, and Blood Institute Growth and Health Study," Journal of Pediatrics, vol. 148, no. 2, pp. 183-187, 2006.

[13] A. M. Albertson, D. L. Franko, D. Thompson et al., "Longitudinal patterns of breakfast eating in black and white adolescent girls," Obesity, vol. 15, no. 9, pp. 2282-2292, 2007.

[14] K. N. Boutelle, J. A. Fulkerson, D. Neumark-Sztainer, M. Story, and S. A. French, "Fast food for family meals: relationships with parent and adolescent food intake, home food availability and weight status," Public Health Nutrition, vol. 10, no. 1, pp. 16-23, 2007.

[15] I. Abete, A. Astrup, J. A. Martínez, I. Thorsdottir, and M. A. Zulet, "Obesity and the metabolic syndrome: role of different dietary macronutrient distribution patterns and specific nutritional components on weight loss and maintenance," Nutrition Reviews, vol. 68, no. 4, pp. 214-231, 2010.

[16] P. R. Deshmukh-Taskar, T. A. Nicklas, C. E. O’Neil, D. R. Keast, J. D. Radcliffe, and S. Cho, "The relationship of breakfast skipping and type of breakfast consumption with nutrient Intake and weight status in children and adolescents: the national health and nutrition examination survey 1999-2006," Journal of the American Dietetic Association, vol. 110, no. 6, pp. 869-878, 2010.

[17] D. Mozaffarian, T. Hao, E. B. Rimm, W. C. Willett, and F. B. $\mathrm{Hu}$, "Changes in diet and lifestyle and long-term weight gain in women and men," The New England Journal of Medicine, vol. 364, no. 25, pp. 2392-2404, 2011.

[18] G. J. Cutler, A. Flood, P. Hannan, and D. Neumark-Sztainer, "Multiple sociodemographic and socioenvironmental characteristics are correlated with major patterns of dietary intake in adolescents," Journal of the American Dietetic Association, vol. 111, no. 2, pp. 230-240, 2011.

[19] N. I. Larson, M. T. Story, and M. C. Nelson, "Neighborhood environments: disparities in access to healthy foods in the U.S," American Journal of Preventive Medicine, vol. 36, no. 1, pp. 74$81,2009$.

[20] M. Scharoun-Lee, J. S. Kaufman, B. M. Popkin, and P. GordonLarsen, "Obesity, race/ethnicity and life course socioeconomic status across the transition from adolescence to adulthood," Journal of Epidemiology and Community Health, vol. 63, no. 2, pp. 133-139, 2009.

[21] M. M. Wall, N. I. Larson, A. Forsyth et al., "Patterns of obesogenic neighborhood features and adolescent overweight: a comparison of statistical approaches," American Journal of Preventive Medicine, vol. 42, no. 5, pp. 65-75, 2012.

[22] M. A. Martin, M. L. Frisco, C. Nau, and K. Burnett, "Social stratification and adolescent overweight in the United States: how income and educational resources matter across families and schools," Social Science Medicine, vol. 74, no. 4, pp. 597606, 2012.

[23] A. F. Leone, S. Ribgy, C. Betterley et al., "Store type and demographic influence on the availability and price of healthful foods, Leon County, Florida," Prevention of Chronic Disease, vol. 8, no. 6, p. A140, 2008.

[24] N. Townsend and C. Foster, "Developing and applying asocioecological model to the promotion of healthy eating in the school," Public Health Nutrition, vol. 13, pp. 1-8, 2011.

[25] B. M. Popkin, L. S. Adair, and S. W. Ng, "Global nutrition transition and the pandemic of obesity in developing countries," Nutrition Reviews, vol. 70, no. 1, pp. 3-21, 2012. 


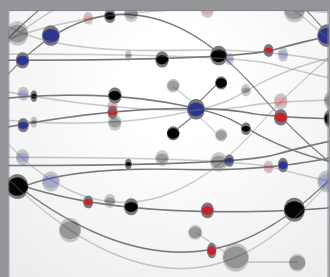

The Scientific World Journal
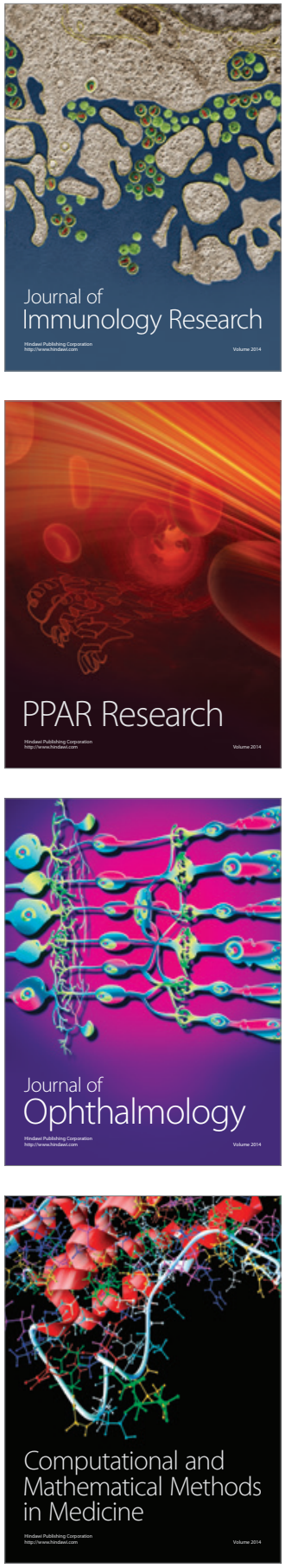

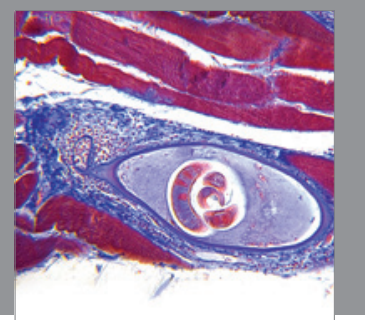

Gastroenterology

Research and Practice
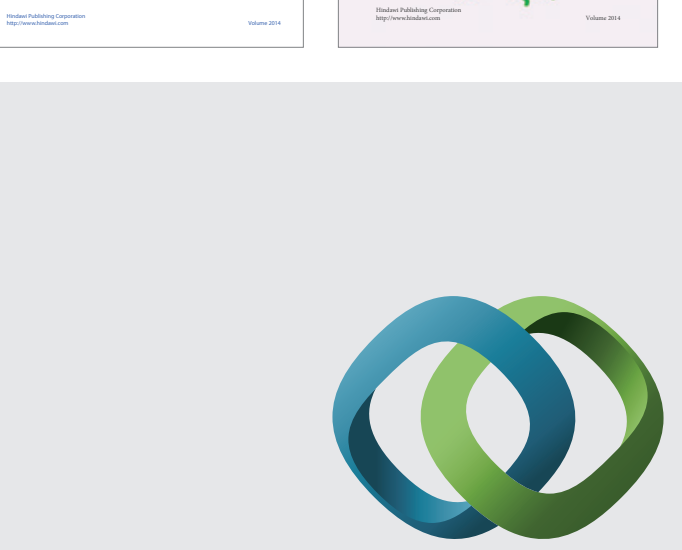

\section{Hindawi}

Submit your manuscripts at

http://www.hindawi.com
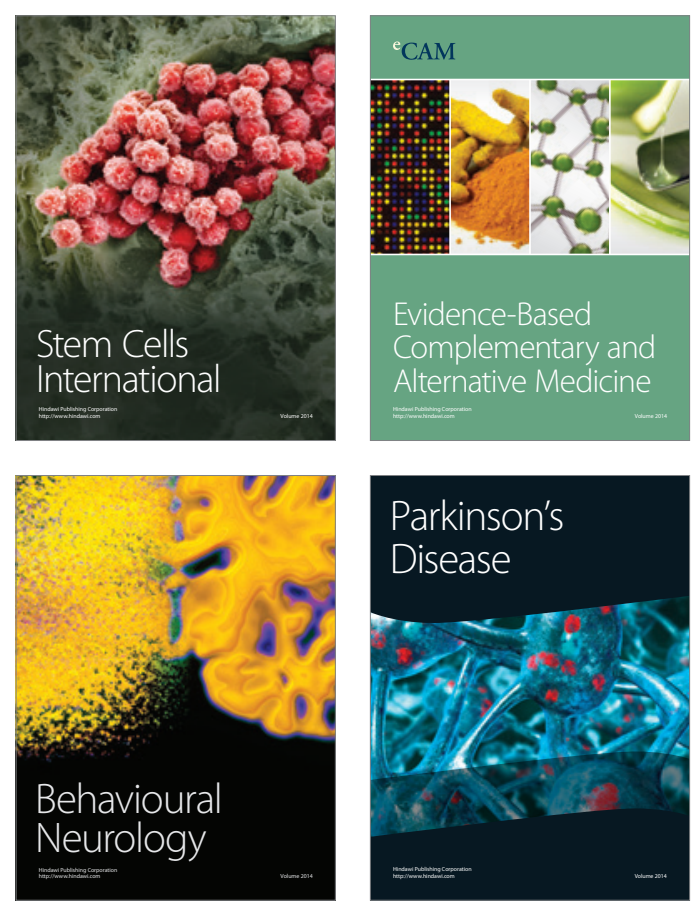

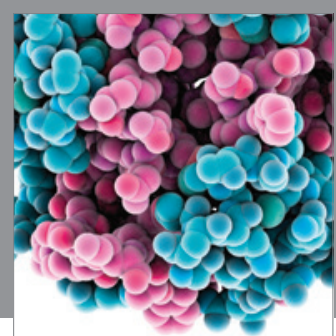

Journal of
Diabetes Research

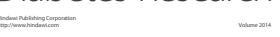

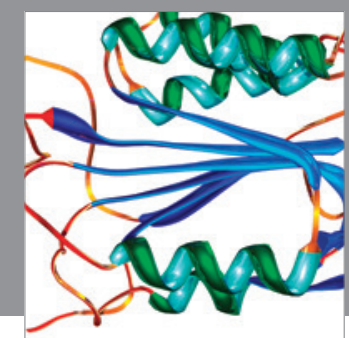

Disease Markers
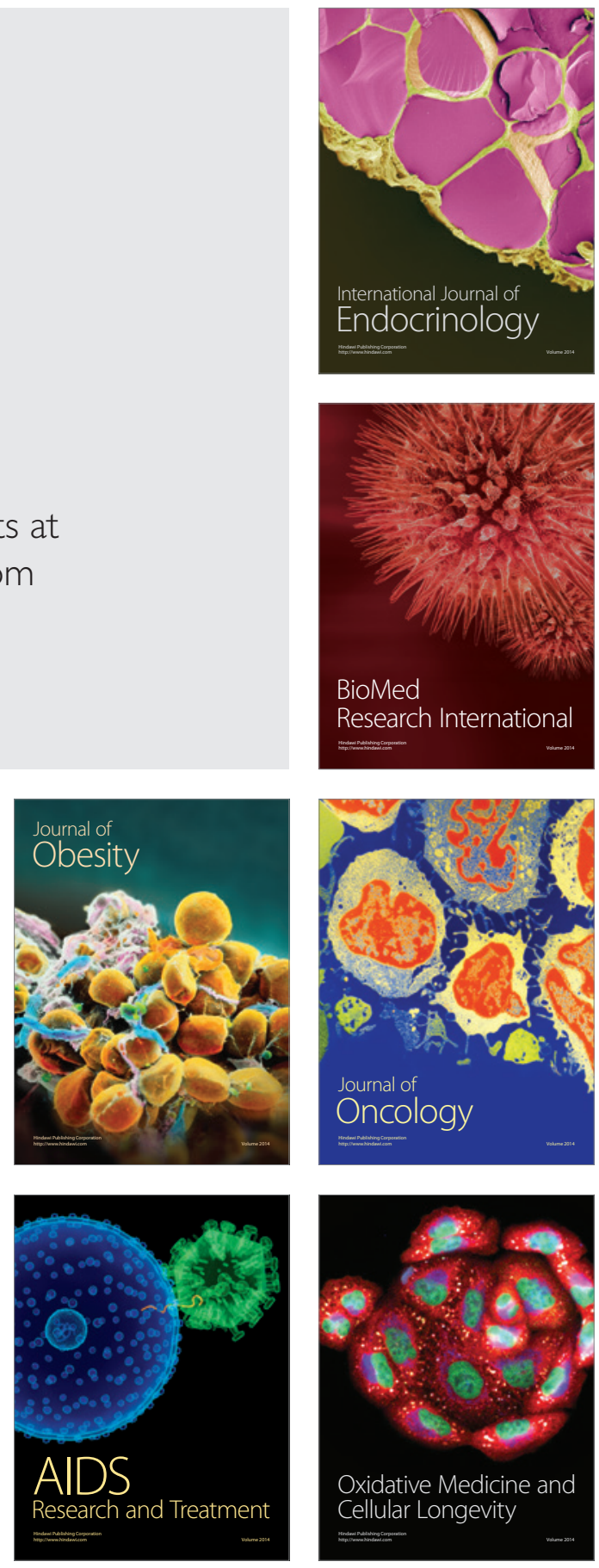